\title{
Applications and Spectral Analysis of some Optimized High Order Low Dispersion and Low Dissipation Schemes
}

\author{
Appanah Rao Appadu* \\ Department of Mathematics and Applied Mathematics, University of Pretoria, Pretoria 0002, South Africa
}

Received: 23 Mar. 2013, Revised: 21 Aug. 2013, Accepted: 21 Aug. 2013

Published online: 1 May. 2014

\begin{abstract}
In Appadu(2012d), we have used the technique of Minimized Integrated Exponential Error for Low Dispersion and Low Dissipation, (MIEELDLD) to construct high order methods with low dispersion and low dissipation properties which approximate the 1D linear advection equation. Modifications to the spatial discretisation schemes constructed by Lockard et al. (1995), Zingg et al. (1996) and Bogey and Bailly (2002) have been obtained and also a modification to the temporal scheme developed by Tam et al. (1993) has been devised. These novel methods obtained using MIEELDLD are more effective in terms of shock-capturing properties as they require less number of points per wavelength than the existing optimized methods for a given accuracy.

In this paper, we perform some numerical experiments dealing with wave propagation with these novel as well as existing, combined spatial and temporal discretisation schemes and compare the variation of two errors namely the Total Mean Square Error and error rate with the CFL. The spectral analysis of two optimized methods made up of spatial discretisation scheme coupled with temporal discretisation scheme is also studied.
\end{abstract}

Keywords: dispersion, dissipation, optimised, high-order, Computational Aeroacoustics

\section{Introduction}

The increasing demand by the aerospace, automotive and other industries for accurate and reliable numerical models of sound generation and propagation in complex physical environments has prompted the construction of new Computational Aeroacoustics (CAA) algorithms. CAA methods have applications which range from aircraft noise to ground vehicle noise to noise from electronic cooling and air-moving devices [9].

In Computational Aeroacoustics (CAA), the accurate prediction of the generation of sound is demanding due to the requirement for preservation of the shape and frequency of wave propagation and generation. It is well-known $[7,14]$ that in order to conduct satisfactory computational aeroacoustics, numerical methods must generate the least possible dispersion and dissipation errors. In general, higher order schemes would be more suitable for CAA than the lower-order schemes since, overall, the former are less dissipative [8]. This is the reason why higher-order spatial discretisation schemes have gained considerable interest in computational aeroacoustics.

\section{Organisation of paper}

This paper is organised as follows. In section 3, we describe briefly the technique of Minimised Integrated Exponential Error for Low Dispersion and Low Dissipation, (MIEELDLD) when used to optimise parameters in numerical methods. In section 4, we describe how the technique of MIEELDLD [5] has been extended to construct low dispersion and low dissipation methods in a Computational Aeroacoustics framework and we list the coefficients of the spatial discretisation methods constructed by Lockard et al. [11], Zingg et al. [16], Bogey and Bailly [6] and also of the temporal discretisation method of Tam et al. [15]. In section 5, we present the results of a numerical experiment dealing with wave propagation and also obtain the variation of the Total Mean Square Error [13] and the error rate vs the

\footnotetext{
*Corresponding author e-mail: Rao.Appadu@up.ac.za, biprao2@yahoo.com
} 
CFL and this helps to obtain a good estimate of the optimal CFL. In section 6, we obtain the variation of the relative phase error vs CFL for two optimised numerical methods. Section 7 includes the conclusion.

\section{The Concept of Minimised Integrated Exponential Error for Low Dispersion and Low Dissipation}

The technique of Minimized Integrated Exponential Error for Low Dispersion and Low Dissipation (MIEELDLD) has been introduced in Appadu and Dauhoo [1]. It basically enables us to choose the optimal parameters from two conditions namely;

(i) small amounts of dissipation when added can help to curb dispersion [10].

(ii) the dissipation and dispersion errors must both be small in a numerical scheme to yield efficient shock-capturing properties.

We now describe the technique of Minimized Integrated Exponential Error for Low Dispersion and Low Dissipation, (MIEELDLD). Suppose the amplification factor of the numerical scheme when applied to the 1-D linear advection equation, given by:

$$
\frac{\partial u}{\partial t}+\beta \frac{\partial u}{\partial x}=0
$$

is

$$
\xi=A+I B .
$$

Then the modulus of the Amplification Factor, $(A F M)$ and the relative phase error, $(R P E)$ are calculated as:

$$
A F M=|\xi|
$$

and

$$
R P E=-\frac{1}{r w} \tan ^{-1} \frac{B}{A},
$$

where $r$ and $w$ are the CFL and phase angle respectively.

The quantities $|1-R P E|$ and $(1-A F M)$ measure the dispersion and dissipation errors respectively.

For a numerical scheme to have low dispersion and low dissipation, we require

$$
|1-R P E|+(1-A F M) \longrightarrow 0
$$

Also when dissipation neutralises dispersion optimally, we have,

$$
|| 1-R P E|-(1-A F M)| \rightarrow 0 \text {. }
$$

Thus on combining these two conditions, we get the following condition necessary for dissipation to neutralise dispersion and for low dispersion and low dissipation character to be satisfied:

$$
\begin{array}{r}
\text { eldld }=|| 1-R P E|-(1-A F M)|+ \\
(|1-R P E|+(1-A F M)) \longrightarrow 0,
\end{array}
$$

where eldld denotes error for low dispersion and low dissipation.

If we now plot the quantity, eldld vs RPE vs AFM, [5], we can see that eldld $=0$ when $R P E=1$ (no dispersion) and $A F M=1$ (no dissipation) and this makes sense. However, the eldld takes a constant value of 2 when $R P E=2$ independent of the value of the AFM and this presents a drawback of the measure. Therefore, we present a modification to the quantity, eldld which is

$$
\begin{array}{r}
\text { eeldld }=\exp (|| 1-R P E|-(1-A F M)|)+ \\
\quad \exp (|1-R P E|+(1-A F M))-2 \longrightarrow 0,
\end{array}
$$

and this quantity goes to zero when $|1-R P E| \rightarrow 0$ and $(1-A F M) \rightarrow 0$.

The eeldld denotes exponential error for low dispersion and low dissipation.

Only one parameter involved

If the CFL is the only parameter, we compute

$$
\int_{0}^{w_{1}} \text { eeldld } d w
$$

for a range of $w \in\left[0, w_{1}\right]$, and this integral will be a function of $r$. The optimal CFL is the one at which the integral quantity is closest to zero.

\section{Two parameters are involved}

Suppose, we now have two parameters, say $\lambda$ and $r$. In that case, we can compute

$$
\int_{0}^{r_{1}} \int_{0}^{w_{1}} \text { eeldld } d w d r
$$

and this integral will be a function of $\lambda$ and we can obtain the optimal value of $\lambda$.

We can also compute

$$
\int_{0}^{w_{1}} \text { eeldld } d w
$$

and this integral will consist of $\lambda$ and $r$. From there, we can obtain the optimal values of both $\lambda$ and $r$.

Considerable and extensive work on the technique of Minimised Integrated Exponential Error for Low Dispersion and Low Dissipation has been carried out in $[1,2,3,4]$.

\section{Extension of MIEELDLD to a CAA framework}

In this section, we describe briefly how the technique of MIEELDLD is used to construct high order, low dispersion and low dissipation spatial schemes, which are 
modifications to the methods constructed by Lockard et al. [11], Zingg et al. [16] and Bogey and Bailly [6].

The spatial derivative proposed by Lockard et al. [11], Bogey and Bailly [6] is approximated as

$$
\frac{\partial u}{\partial x} \approx \frac{1}{h} \sum_{i=-N}^{N} a_{i} u(x+i h),
$$

and the coefficients are listed in Table (1).

In the case of the spatial discretisation proposed by Zingg et al. [16], we have

$$
\begin{array}{r}
\frac{\partial u}{\partial x}=\frac{1}{h} \sum_{i=1}^{3} a_{i}\left(u\left(x_{0}+i h\right)-u\left(x_{0}-i h\right)\right)+ \\
\frac{1}{h}\left(d_{0} u\left(x_{0}\right)+\sum_{i=1}^{3} d_{i}\left(u\left(x_{0}+i h\right)+u\left(x_{0}-i h\right)\right)\right) .
\end{array}
$$

To simplify things, we abbreviate the spatial schemes constructed by Lockard et al. [11], Zingg et al. [16], Bogey and Bailly [6] as Lockard, Zingg and Bogey. Using MIEELDLD, we have proposed modification to the existing schemes described by Eqs. (6) and (7) and Table (1). The coefficients of the modified schemes are shown in Table (2). For more details on how the novel spatial methods have been constructed, please refer to [5].

The temporal discretisation scheme proposed by Tam et al. [15] is given as follows:

$$
u^{n+1}-u^{n} \approx k \sum_{i=0}^{3} b_{i}\left(\frac{d U}{d t}\right)^{n-i}
$$

where $b_{0}=2.30255809, \quad b_{1}=-2.49100760$, $b_{2}=1.5743093$ and $b_{3}=-0.38589142$.

Using MIEELDLD, a modification to the temporal discretisation scheme proposed by Tam et al. [15] has been obtained and is termed as Tam-Modified. We refer to Eq. (8) with the coefficients being; $b_{0}=2.27963782$, $b_{1}=-2.42224680, \quad b_{2}=1.50558014$ and $b_{3}=-0.36297116$.

Hence, we have three new spatial discretisation schemes and one new temporal discretisation method. In [5], we have obtained the range of stability of the methods namely: Zingg, Lockard, and Bogey, when they are combined with the temporal discretisation scheme of Tam et al. [15]. Thus, we instance in the case of the spatial scheme of Lockard et al. [11] coupled with the temporal scheme of Tam et al. [15], this combined method is called Lockard + Tam. The same type of abbreviation is used for the remaining optimised methods.

In [5], we have obtained the range of stability of the novel methods: Zingg-New, Lockard-New and Bogey-New when they are combined with the temporal discretisation scheme, Tam-Modified. Table (3) summarises details for the stability of the six finite difference schemes.
Table 1: Coefficients for spatial discretisation schemes derived by Zingg et al. [16], Lockard et al. [11] and Bogey and Bailly [6]

\begin{tabular}{c|c|c}
\hline ZINGG & LOCKARD & BOGEY \\
\hline \hline$a_{1}=0.759961$ & $a_{-4}=0.010393$ & $a_{0}=0$ \\
\hline$a_{2}=-0.158122$ & $a_{-3}=-0.084670$ & $a_{1}=0.841570$ \\
\hline$a_{3}=0.018761$ & $a_{-2}=0.342031$ & $a_{2}=0.244679$ \\
\hline$d_{0}=0.1$ & $a_{-1}=-1.052681$ & $a_{3}=0.059464$ \\
\hline$d_{1}=-0.076385$ & $a_{0}=0.287274$ & $a_{4}=-0.007651$ \\
\hline$d_{2}=0.032290$ & $a_{1}=0.586162$ & $a_{-1}=-0.841570$ \\
\hline$d_{3}=-0.005905$ & $a_{2}=-0.098144$ & $a_{-2}=-0.244679$ \\
\hline- & $a_{3}=0.009662$ & $a_{-3}=-0.059464$ \\
\hline
\end{tabular}

Table 2: Coefficients for new spatial discretisation schemes using MIEELDLD which are modifications to the schemes designed by Zingg et al. [16], Lockard et al. [11], and Bogey and Bailly [6] (to 6 d.p)

\begin{tabular}{lll}
\hline Zingg-New & Lockard-New & Bogey-New \\
\hline$a_{1}=0.764316$ & $a_{-4}=0.011346$ & $a_{0}=0$ \\
\hline$a_{2}=-0.161452$ & $a_{-3}=-0.089198$ & $a_{1}=0.844367$ \\
\hline$a_{3}=0.019530$ & $a_{-2}=0.349998$ & $a_{2}=-0.248033$ \\
\hline$d_{0}=0.1$ & $a_{-1}=-1.058267$ & $a_{3}=0.061300$ \\
\hline$d_{1}=-0.076438$ & $a_{0}=0.286601$ & $a_{4}=-0.008050$ \\
\hline$d_{2}=0.032300$ & $a_{1}=0.589520$ & $a_{-1}=-0.844367$ \\
\hline$d_{3}=-0.005863$ & $a_{2}=-0.1$ & $a_{-2}=-0.248033$ \\
\hline- & $a_{3}=0.01$ & $a_{-3}=-0.061300$ \\
\hline- & - & $a_{-4}=0.008050$ \\
\hline
\end{tabular}

Table 3: Region of stability for some combined spatialtemporal discretisation schemes

\begin{tabular}{lll}
\hline Spatial scheme & Temporal scheme & max. value of $r$ \\
\hline Zingg & Tam & 0.23 \\
\hline Zingg-New & Tam-Modified & 0.24 \\
\hline Lockard & Tam & 0.20 \\
\hline Lockard-New & Tam-Modified & 0.22 \\
\hline Bogey & Tam & 0.20 \\
\hline Bogey-New & Tam-Modified & 0.21 \\
\hline
\end{tabular}

\section{Numerical Results}

The test problem we consider is the propagation of the Boxcar function [12] subject to $\frac{\partial u}{\partial t}+\frac{\partial u}{\partial x}=0$. This test problem involves discontinuous initial conditions. The 
initial disturbance can be written as $g(x)=H(x+25)-H(x-25)$, for $0 \leq x \leq 450$, where $H(x)$ is the Heaviside function.

To evaluate the solution accuracy, we define the error vector as $\vec{E}=\left(E_{1}, \ldots, E_{N}\right)^{T}$, where $E_{i}=U\left(x_{i}\right)-u_{i}, 1 \leq$ $i \leq N, U\left(x_{i}\right)$ is the exact solution at the point $x_{i}$, and $u_{i}$ is the numerical solution at the point $x_{i}$ and $N$ is the number of grid points. We compute the Total Mean Square Error, $E_{T}$ at the different CFL:0.025, 0.05, 0.08, 0.10, 0.125, 0.16 and 0.20 as

$$
E_{T}=\frac{\sum_{i=1}^{N}\left(U\left(x_{i}\right)-u_{i}\right)^{2}}{N}
$$

The Total Mean Square Error has been shown to be a measure of the sum of the dispersion and dissipation errors [13].

The error rate, enum computed as

$$
e_{\text {num }}=\left(\frac{\sum_{i=1}^{N}\left(U\left(x_{i}\right)-u_{i}\right)^{2}}{\sum_{i=1}^{N}\left(U\left(x_{i}\right)\right)^{2}}\right)^{1 / 2},
$$

is also compared for each scheme at some values of CFL.

Table 4: Errors for boxcar propagation at dimensionless time, $t=400$ at some values of CFL using spatial scheme of Lockard et al. [11] coupled with temporal scheme of Tam et al. [15]

\begin{tabular}{lll}
\hline CFL & Total Mean Square Error & error rate \\
\hline 0.025 & $1.4563 \times 10^{-3}$ & 0.1166 \\
\hline 0.05 & $1.4246 \times 10^{-3}$ & 0.1153 \\
\hline 0.08 & $1.4191 \times 10^{-3}$ & 0.1151 \\
\hline 0.10 & $1.3687 \times 10^{-3}$ & 0.1130 \\
\hline 0.125 & $1.3447 \times 10^{-3}$ & 0.1120 \\
\hline 0.16 & $1.3074 \times 10^{-3}$ & 0.1105 \\
\hline 0.20 & $1.3114 \times 10^{-3}$ & 0.1063 \\
\hline
\end{tabular}

The results of the boxcar propagation are shown in Figs. (1) to (3).

The Total Mean Square Error and error rate of the six schemes at some different values of CFL are tabulated in Tables (4) to (9). We can deduce that the scheme Lockard+Tam and Lockard-New+Tam-Modified are most effective at CFL 0.20. Fig. (4) shows that the new scheme has smaller total errors at a given CFL. Also, with increase in CFL, the general trend is that the errors decrease.

Both the schemes Zingg+Tam and Zingg-New+Tam-Modified are more effective at low CFL as can be deduced from Fig. (5). Also, the new scheme is
Table 5: Errors for boxcar propagation at dimensionless time, $t=400$ at some values of CFL using spatial scheme Lockard-New coupled with temporal scheme Tam-Modified

\begin{tabular}{lll}
\hline CFL & Total Mean Square Error & error rate \\
\hline 0.025 & $1.3951 \times 10^{-3}$ & 0.1141 \\
\hline 0.05 & $1.3622 \times 10^{-3}$ & 0.1128 \\
\hline 0.08 & $1.3566 \times 10^{-3}$ & 0.1252 \\
\hline 0.10 & $1.3053 \times 10^{-3}$ & 0.1038 \\
\hline 0.125 & $1.2821 \times 10^{-3}$ & 0.1094 \\
\hline 0.16 & $1.2491 \times 10^{-3}$ & 0.1080 \\
\hline 0.20 & $1.2598 \times 10^{-3}$ & 0.1084 \\
\hline
\end{tabular}

Table 6: Errors for boxcar propagation at dimensionless time, $t=400$ at some values of CFL using spatial scheme of Zingg et al [16] coupled with temporal scheme of Tam et al. [15]

\begin{tabular}{lll}
\hline CFL & Total Mean Square Error & error rate \\
\hline 0.025 & $1.5121 \times 10^{-3}$ & 0.1188 \\
\hline 0.05 & $1.5378 \times 10^{-3}$ & 0.1198 \\
\hline 0.08 & $1.5410 \times 10^{-3}$ & 0.1199 \\
\hline 0.10 & $1.5998 \times 10^{-3}$ & 0.1222 \\
\hline 0.125 & $1.6383 \times 10^{-3}$ & 0.1237 \\
\hline 0.16 & $1.7499 \times 10^{-3}$ & 0.1278 \\
\hline 0.16 & $1.8033 \times 10^{-3}$ & 0.1297 \\
\hline
\end{tabular}

Table 7: Errors for boxcar propagation at dimensionless time, $t=400$ at some values of CFL using spatial scheme Zingg-New coupled with temporal scheme Tam-Modified

\begin{tabular}{lll}
\hline CFL & Total Mean Square Error & error rate \\
\hline 0.025 & $1.3636 \times 10^{-3}$ & 0.1128 \\
\hline 0.05 & $1.3814 \times 10^{-3}$ & 0.1135 \\
\hline 0.08 & $1.3846 \times 10^{-3}$ & 0.1137 \\
\hline 0.10 & $1.4269 \times 10^{-3}$ & 0.1154 \\
\hline 0.125 & $1.4564 \times 10^{-3}$ & 0.1166 \\
\hline 0.16 & $1.5453 \times 10^{-3}$ & 0.1201 \\
\hline 0.20 & $1.5884 \times 10^{-3}$ & 0.1218 \\
\hline
\end{tabular}

more effective at a given CFL.

Fig. (6) shows the variation of the Total Mean Square Error vs the CFL and for both schemes namely Bogey+Tam and Bogey-New+Tam-Modified. The errors decrease with increase in CFL. Moreover, the new 
Table 8: Errors for boxcar propagation at dimensionless time, $t=400$ at some values of CFL using spatial scheme Bogey and Bailly coupled with temporal scheme Tam et al. [15]

\begin{tabular}{lll}
\hline CFL & Total Mean Square Error & error rate \\
\hline 0.025 & $1.5220 \times 10^{-3}$ & 0.1192 \\
\hline 0.05 & $1.4541 \times 10^{-3}$ & 0.1165 \\
\hline 0.08 & $1.4083 \times 10^{-3}$ & 0.1147 \\
\hline 0.10 & $1.3401 \times 10^{-3}$ & 0.1118 \\
\hline 0.125 & $1.2895 \times 10^{-3}$ & 0.1097 \\
\hline 0.16 & $1.2341 \times 10^{-3}$ & 0.1073 \\
\hline 0.20 & $1.2197 \times 10^{-3}$ & 0.1067 \\
\hline
\end{tabular}

Table 9: Errors for boxcar propagation at dimensionless time, $t=400$ at some values of CFL using spatial scheme Bogey-New coupled with temporal scheme Tam-Modified

\begin{tabular}{lll}
\hline CFL & Total Mean Square Error & error rate \\
\hline 0.025 & $1.4426 \times 10^{-3}$ & 0.1160 \\
\hline 0.05 & $1.3721 \times 10^{-3}$ & 0.1132 \\
\hline 0.08 & $1.3164 \times 10^{-3}$ & 0.1108 \\
\hline 0.10 & $1.2401 \times 10^{-3}$ & 0.1076 \\
\hline 0.125 & $1.1848 \times 10^{-3}$ & 0.1052 \\
\hline 0.16 & $1.1577 \times 10^{-3}$ & 0.1040 \\
\hline 0.20 & $1.2054 \times 10^{-3}$ & 0.1061 \\
\hline
\end{tabular}

scheme is more effective at a given CFL.

\section{Dispersive and dissipative properties of two high-order numerical schemes}

In this section, we study the dissipative and dispersive properties of two methods and also attempt to locate the optimal CFL. Single expressions for the two methods namely: spatial discretisation of Bogey and Bailly [6] coupled with the temporal discretisation scheme of Tam et al. [15] abbreviated as Bogey + Tam and our novel method, abbreviated as Bogey-New + Tam-Modified are given as follows:

$$
\begin{array}{r}
u_{i}^{n+4}=u_{i}^{n+3}-r\left(T _ { 1 } \left(S_{1}\left(u_{i+1}^{n+3}-u_{i-1}^{n+3}\right)-S_{2}\left(u_{i+2}^{n+3}-u_{i-2}^{n+3}\right)\right.\right. \\
\left.+S_{3}\left(u_{i+3}^{n+3}-u_{i-3}^{n+3}\right)\right)+T_{2}\left(S_{1}\left(u_{i+1}^{n+2}-u_{i-1}^{n+2}\right)-\right. \\
\left.S_{2}\left(u_{i+2}^{n+2}-u_{i-2}^{n+2}\right)+S_{3}\left(u_{i+3}^{n+2}-u_{i-3}^{n+2}\right)\right) \\
+T_{3}\left(S_{1}\left(u_{i+1}^{n+1}-u_{i-1}^{n+1}\right)-S_{2}\left(u_{i+2}^{n+1}-u_{i-2}^{n+1}\right)\right. \\
\left.+S_{3}\left(u_{i+3}^{n+1}-u_{i-3}^{n+1}\right)\right)+T_{4}\left(S_{1}\left(u_{i+1}^{n}-u_{i-1}^{n}\right)\right. \\
\left.\left.-S_{2}\left(u_{i+2}^{n}-u_{i-2}^{n}\right)+S_{3}\left(u_{i+3}^{n}-u_{i-3}^{n}\right)\right)\right),
\end{array}
$$

In the case of Bogey+Tam, we have $T_{1}=2.30255809$, $T_{2}=-2.49100760, \quad T_{3}=1.57434093$, $T_{4}=-0.38589142, \quad S_{1}=0.77088238$, $S_{2}=-0.16670590, S_{3}=0.02084314$ while in the case of Bogey-New + Tam-Modified, we have $T_{1}=2.27963782$, $T_{2}=-2.42224680, \quad T_{3}=1.50558014$, $T_{4}=-0.36297115, \quad S_{1}=0.76772067$, $S_{2}=-0.16417654, S_{3}=0.02021080$.

The amplification factor of the Bogey+Tam method satisfies the following complicated equation:

$$
\begin{aligned}
\xi^{4}=\xi^{3}-r & \left(2.30255809 A_{1} \xi^{3}-2.49100760 A_{1} \xi^{2}\right. \\
& \left.+1.57434093 A_{1} \xi-0.38589142 A_{1}\right)
\end{aligned}
$$

where

$$
\begin{array}{r}
A_{1}=2 I(0.8415701254 \sin (w)- \\
-0.2446786317 \sin (2 w) \\
+0.0594635848 \sin (3 w) \\
-0.0076509041 \sin (4 w)) .
\end{array}
$$

Therefore, it is not possible to obtain $\xi$ in terms of $r$ and $w$ so that the expressions for the $A F M$ and $R P E$ cannot be expressed in terms of $r$ and $w$.

So, we adopt an approach where we fix the CFL number and the phase angle, $w$. For instance, we compute the $A F M$ and $R P E$ at a given CFL for some different phase angles, $w$ say $0, \pi / 8, \pi / 4, \pi / 2,3 \pi / 8, \pi / 2,5 \pi / 8,3 \pi / 4$, $7 \pi / 8$ and $\pi$. Then, we repeat the whole procedure for two other $C F L$ numbers, say 0.1 and 0.2 .

For instance for the case, $r=0.025$ and $w=\pi / 8$, we have four values for $\xi$ namely; $\xi_{1}=0.999518200-0.009816207782 \quad I$, $\xi_{2}=0.02398334562+0.1517460053 \quad I$, $\xi_{3}=-0.1468577715-0.1047185759 \quad I \quad$ and $\xi_{4}=0.1229226059-0.05981397341$. In that case, we choose the physical mode and obtain the $A F M=\left|\xi_{1}\right|=1$ and $R P E=\frac{-1}{0.025 .(\pi / 8)} \arg \left(\xi_{1}\right)=0.9998867719$.

Table (10) show some values of $A F M$ and $R P E$ at three different values of CFL obtained at some values of the phase angle for the Bogey+Tam method. It is seen that the $A F M$ is almost one and is almost independent of the CFL and the phase angle but such is not the case with the 


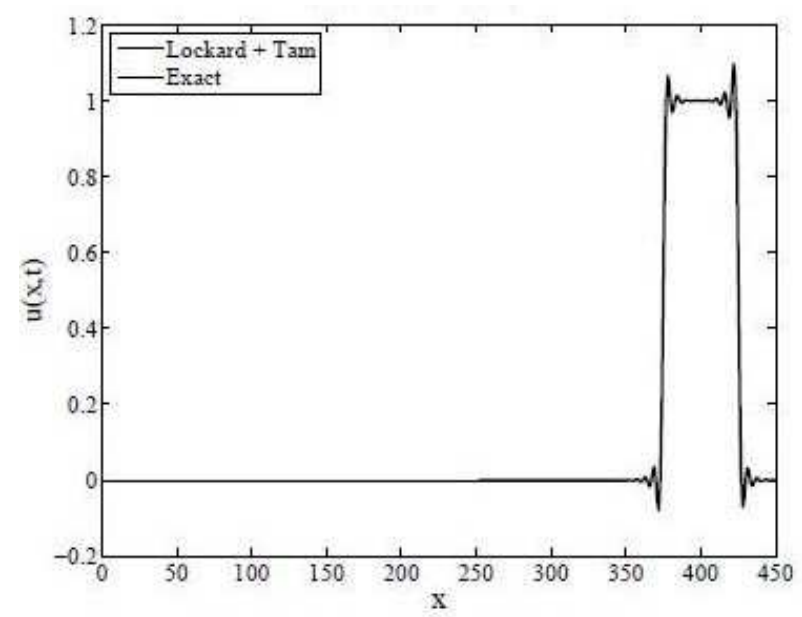

(a) Lockard + Tam

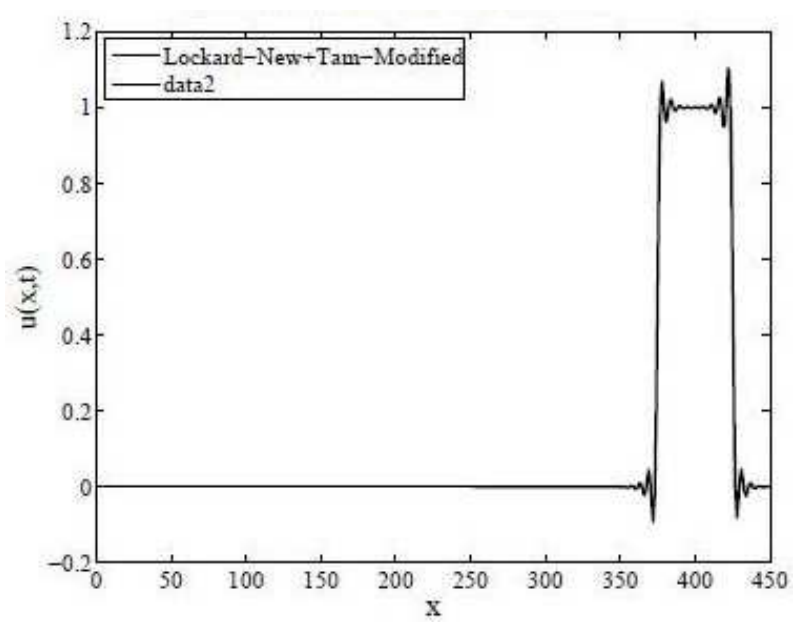

(b) Lockard-New + Tam-Modified

Fig. 1: Results of Boxcar propagation at dimensionless time, $t=400$ at CFL 0.025 .

$R P E$. The general trend with the RPE is that as the phase angle increases, it decreases for all the three values of CFL considered hence exhibiting phase lag behaviour. Table (11) shows the values of the $A F M$ and $R P E$ for our new optimised method which is a modification of the spatial scheme of Bogey and Bailly [6] coupled with temporal scheme of Tam and al. [15]. Again, the same observation is made i.e. the $A F M$ is mostly one and is almost independent of the CFL and phase angle.

Fig. (7) shows the plots of the relative phase error vs the phase angle, $w \in[0, \pi / 2]$ for the two methods namely Bogey+Tam and Bogey-New+Tam-Modified at three different CFL namely $0.025,0.1$ and 0.2 . Mainly phase lag behaviour is observed. Our new scheme has better dispersion properties for all the three different values of CFL considered.

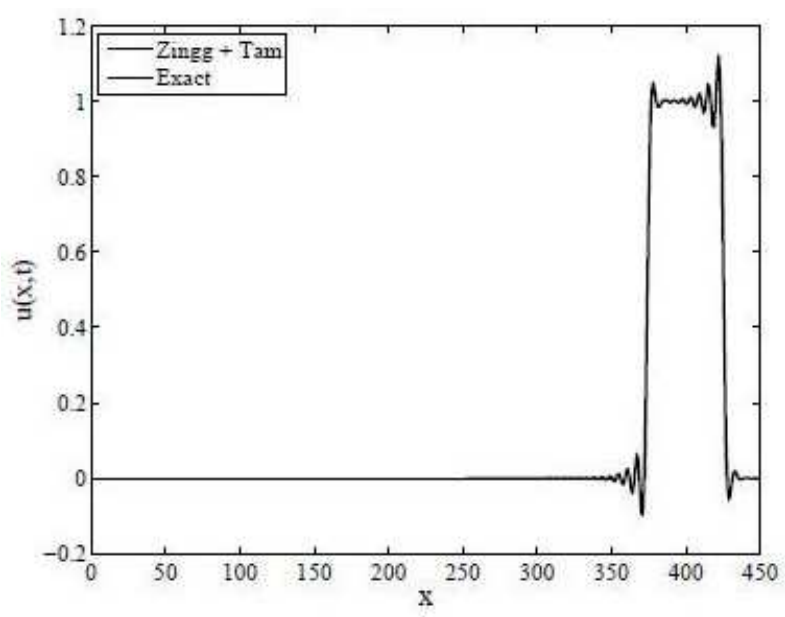

(a) Zingg+Tam

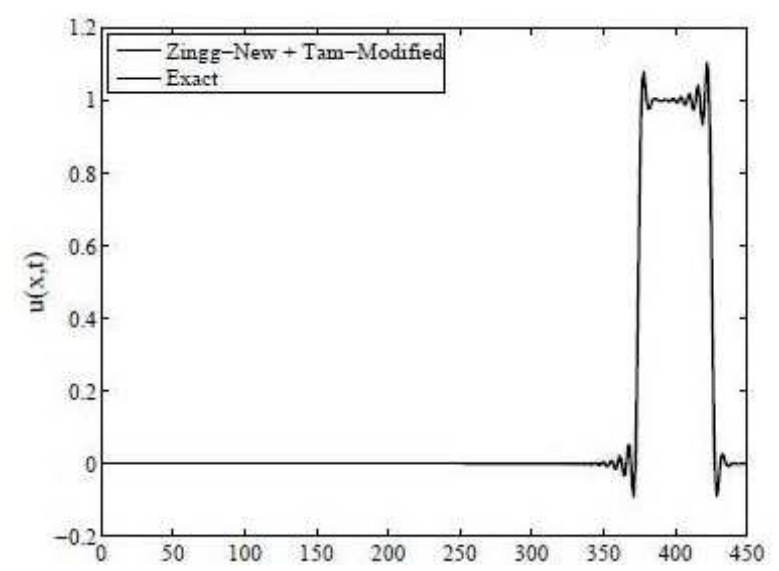

(b) Zingg-New + Tam-Modified

Fig. 2: Results of Boxcar propagation at dimensionless time, $t=400$ at CFL 0.025 .

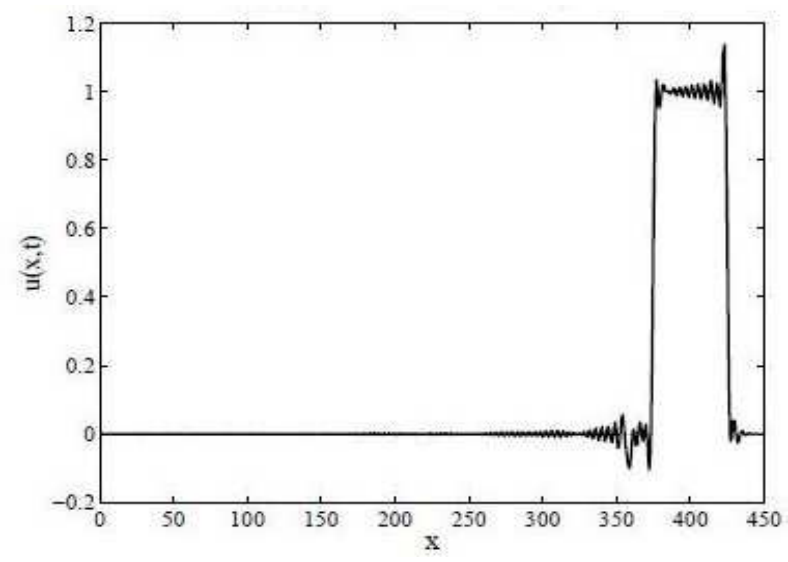

(a) Bogey + Tam at CFL 0.20 


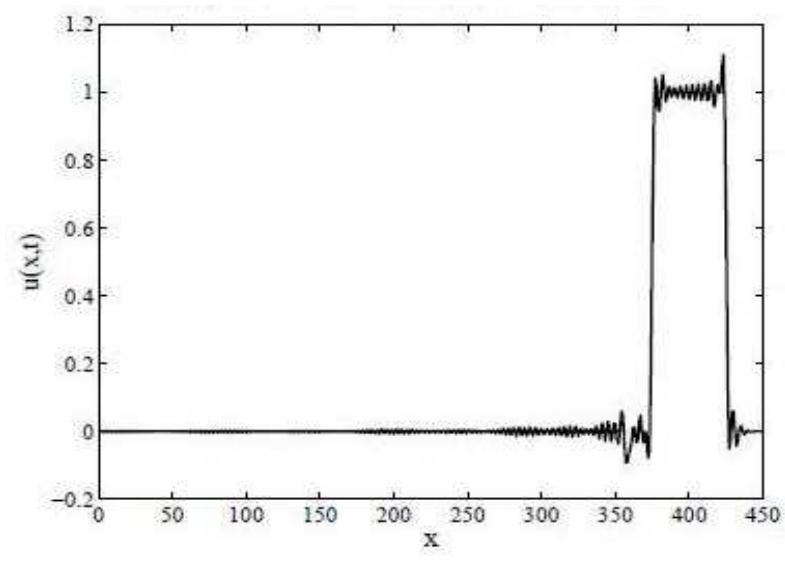

(b) Bogey-New + Tam-Modified at CFL 0.16

Fig. 3: Results of Boxcar propagation at dimensionless time, $t=400$.

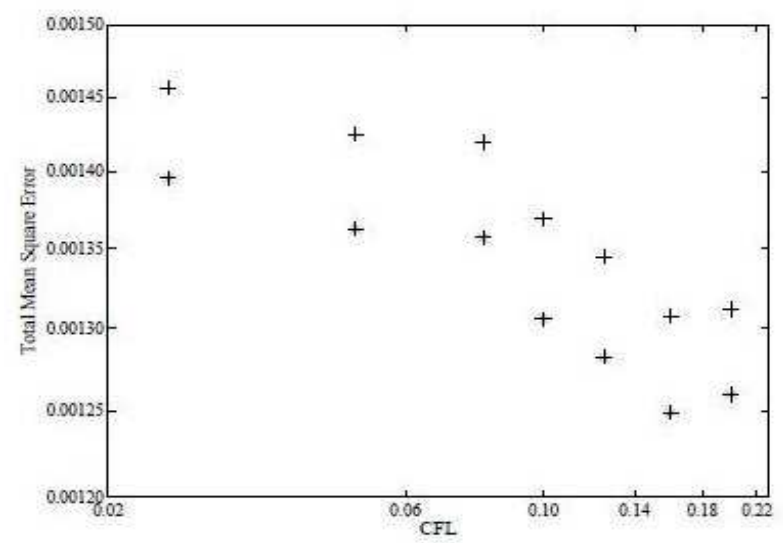

Fig. 4: Plot of Total Mean Square Error vs CFL number for Lockard+Tam '+' and Lockard-New+Tam-Modified '*'.

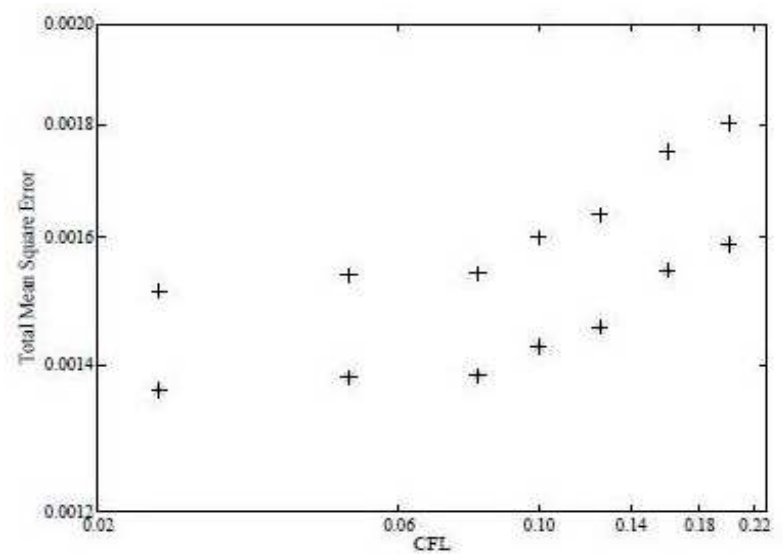

Fig. 5: Plot of Total Mean Square Error vs CFL number for Zingg+Tam '+' and Zingg-New+Tam-Modified '*'.

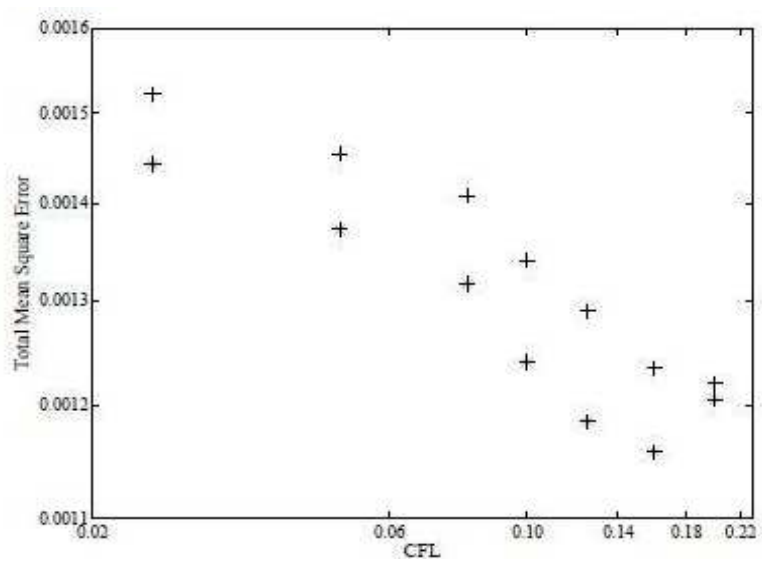

Fig. 6: Plot of Total Mean Square Error vs CFL number for Bogey+Tam '+' and Bogey-New+Tam-Modified '*'.

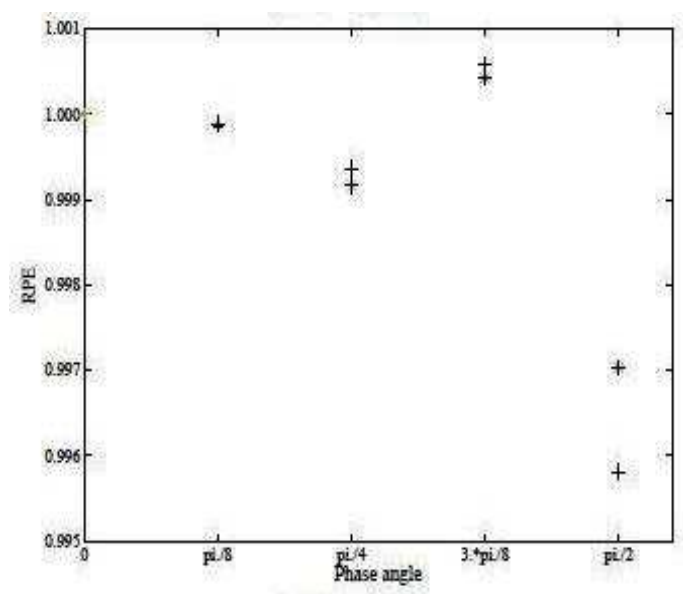

(a) $\mathrm{CFL}=0.025$

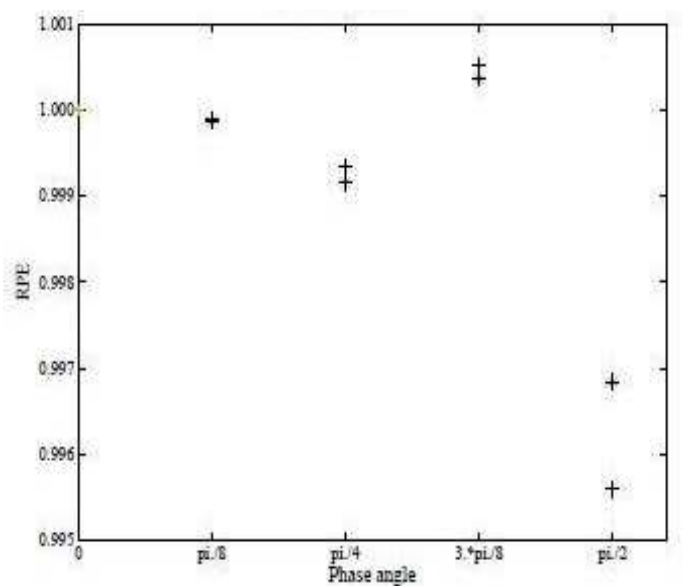

(b) $\mathrm{CFL}=0.1$ 


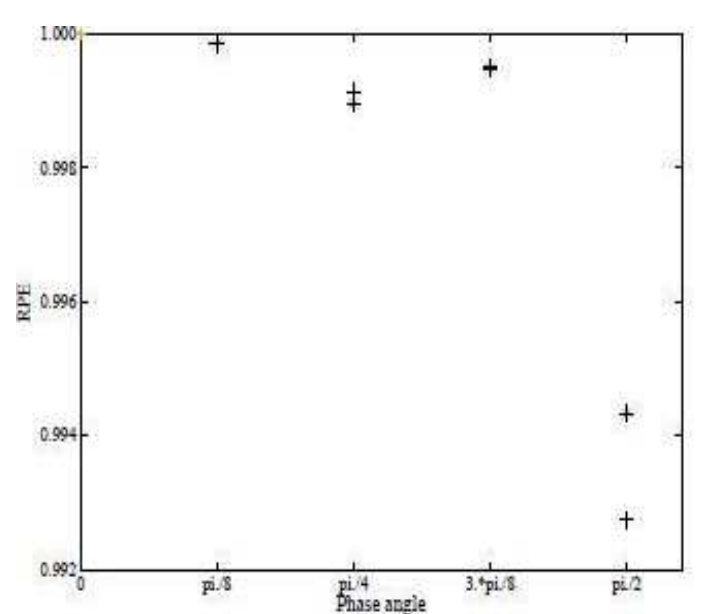

(c) $\mathrm{CFL}=0.2$

Fig. 7: Plot of the RPE vs phase angle for two methods: Bogey+Tam $(+)$ and Bogey-New + Tam-Modified $(*)$ at three different values of CFL.

\section{Conclusion}

In this work, we have used new spatial discretisation schemes and the novel temporal discretisation method constructed in [5] to solve a 1-D wave propagation experiment. We have obtained the variation of the Total Mean Square Error and error rate vs the CFL for six numerical methods. It is seen that our new schemes are more efficient. Also, we have done some spectral analysis of two methods: Bogey and Bailly [6] coupled with temporal scheme of Tam et al. [15] and also that of Bogey-New combined with Tam-Modified and deduced that the shock-capturing property of Bogey-New combined with Tam-Modified is in general better.

\section{Nomenclature}

$I=\sqrt{ }(-1)$

$k$ : time step

$h$ : spatial step

$n$ : time level

$\beta$ : advection velocity

$r$ : CFL or Courant number

$r=\frac{\beta k}{h}$

$w$ : phase angle in 1-D

$w=\theta h$

$R P E$ : relative phase error per unit time step

$A F M$ : modulus of amplification factor

LDLD: Low Dispersion and Low Dissipation

IEELDLD: Integrated Exponential Error for Low Dispersion and Low Dissipation

MIEELDLD: Minimised Integrated Exponential Error
Table 10: Some values for $A F M$ and RPE at CFL 0.025, 0.1 and 0.20 for the Bogey + Tam method.

\begin{tabular}{|c|c|c|c|}
\hline Phase angle & CFL & $A F M$ & $R P E$ \\
\hline 0 & 0.025 & 1 & 1 \\
\hline$\pi / 8$ & 0.025 & 1 & 0.999887 \\
\hline$\pi / 4$ & 0.025 & 1.000000 & 0.999362 \\
\hline $3 \pi / 8$ & 0.025 & 1.000000 & 1.000580 \\
\hline$\pi / 2$ & 0.025 & 1.000000 & 0.995808 \\
\hline $5 \pi / 8$ & 0.025 & 1.000000 & 0.937222 \\
\hline $3 \pi / 4$ & 0.025 & 1.000000 & 0.748499 \\
\hline $7 \pi / 8$ & 0.025 & 1.000000 & 0.405732 \\
\hline$\pi$ & 0.025 & 1 & 0 \\
\hline 0 & 0.1 & 1 & 1 \\
\hline$\pi / 8$ & 0.1 & 1.000000 & 0.999886 \\
\hline$\pi / 4$ & 0.1 & 1.000000 & 0.999348 \\
\hline $3 \pi / 8$ & 0.1 & 1.000001 & 1.000510 \\
\hline$\pi / 2$ & 0.1 & 0.999998 & 0.995595 \\
\hline $5 \pi / 8$ & 0.1 & 0.999991 & 0.936843 \\
\hline $3 \pi / 4$ & 0.1 & 0.999994 & 0.748243 \\
\hline $7 \pi / 8$ & 0.1 & 1.000001 & 0.405709 \\
\hline$\pi$ & 0.1 & 1 & 0 \\
\hline 0 & 0.2 & 1 & 1 \\
\hline$\pi / 8$ & 0.2 & 1.000000 & 0.999873 \\
\hline$\pi / 4$ & 0.2 & 0.999998 & 0.999144 \\
\hline $3 \pi / 8$ & 0.2 & 0.999940 & 0.999520 \\
\hline$\pi / 2$ & 0.2 & 0.999612 & 0.992735 \\
\hline $5 \pi / 8$ & 0.2 & 0.998945 & 0.931984 \\
\hline $3 \pi / 4$ & 0.2 & 0.999185 & 0.744911 \\
\hline $7 \pi / 8$ & 0.2 & 0.999960 & 0.405384 \\
\hline$\pi$ & 0.2 & 1 & 0 \\
\hline
\end{tabular}

for Low Dispersion and Low Dissipation.

\section{Acknowledgement}

This work was funded through the Research Development Programme of the University of Pretoria. The period of funding is from January 2012 to January 2014. 
Table 11: Some values for $A F M, R P E$ at CFL $0.025,0.10$ and 0.20 for the Bogey-New + Tam-Modified method.

\begin{tabular}{|c|c|c|c|}
\hline Phase angle & CFL & $A F M$ & $R P E$ \\
\hline 0 & 0.025 & 1 & 1 \\
\hline$\pi / 8$ & 0.025 & 1.000000 & 0.999864 \\
\hline$\pi / 4$ & 0.025 & 1.000000 & 0.999161 \\
\hline $3 \pi / 8$ & 0.025 & 1.000000 & 1.000424 \\
\hline$\pi / 2$ & 0.025 & 1.000000 & 0.997031 \\
\hline $5 \pi / 8$ & 0.025 & 1.000000 & 0.941147 \\
\hline $3 \pi / 4$ & 0.025 & 1.000000 & 0.754127 \\
\hline $7 \pi / 8$ & 0.025 & 1.000000 & 0.409761 \\
\hline$\pi$ & 0.025 & 1 & 0 \\
\hline 0 & 0.025 & 1 & 1 \\
\hline$\pi / 8$ & 0.1 & 1.000000 & 0.999864 \\
\hline$\pi / 4$ & 0.1 & 0.999999 & 0.999149 \\
\hline $3 \pi / 8$ & 0.1 & 0.999996 & 1.000363 \\
\hline$\pi / 2$ & 0.1 & 0.999985 & 0.996843 \\
\hline $5 \pi / 8$ & 0.1 & 0.999962 & 0.940809 \\
\hline $3 \pi / 4$ & 0.1 & 0.999972 & 0.753895 \\
\hline $7 \pi / 8$ & 0.1 & 0.999997 & 0.409740 \\
\hline$\pi$ & 0.1 & 1 & 0 \\
\hline 0 & 0.2 & 1 & 1 \\
\hline$\pi / 8$ & 0.2 & 0.999999 & 0.999852 \\
\hline$\pi / 4$ & 0.2 & 0.999991 & 0.998971 \\
\hline $3 \pi / 8$ & 0.2 & 0.999878 & 0.999498 \\
\hline$\pi / 2$ & 0.2 & 0.999434 & 0.994332 \\
\hline $5 \pi / 8$ & 0.2 & 0.998608 & 0.936486 \\
\hline $3 \pi / 4$ & 0.2 & 0.998875 & 0.750885 \\
\hline $7 \pi / 8$ & 0.2 & 0.999904 & 0.409443 \\
\hline$\pi$ & 0.2 & 1 & 0 \\
\hline
\end{tabular}

\section{References}

[1] A. R. Appadu and M. Z. Dauhoo, Some Applications of the Concept of Minimized Integrated Exponential Error for Low Dispersion and Low Dissipation Schemes, International Journal for Numerical Methods in Fluids, 65, 578-601 (2011).

[2] A. R. Appadu, Some Applications of the Concept of Minimized Integrated Exponential Error for Low Dispersion and Low Dissipation Schemes, International Journal for Numerical Methods in Fluids, 68, 244-268 (2012).

[3] A. R. Appadu, Investigating the Shock-Capturing Properties of Some Composite Numerical Schemes for the 1-D Linear
Advection Equation, International Journal of Computer Applications in Technology, 43, 79-92 (2012).

[4] A. R. Appadu, Comparison of Some Optimisation techniques for Numerical Schemes Discretising Equations with Advection Terms, International Journal of Innovative Computing and Applications, 4, 12-27 (2012).

[5] A. R. Appadu, The Technique of MIEELDLD in Computational Aeroacoustics, Journal of Applied Mathematics, 2012, Article ID 783101, 30 pages,

[6] C. Bogey and C. Bailly, A Family of Low Dispersive and Low Dissipative Explicit Schemes for Computing the Aerodynamic Noise, 2002-2509 (2002).

[7] J. Hardin and M. Y. Hussaini, Computational Aeroacoustics, Springer-Verlag, New-York, Berlin, (1992).

[8] R. Hixon, Evaluation of High-Accuracy MacCormack-Type Scheme Using Benchmark Problems, NASA Contractor Report 202324, (1997).

[9] Konstantin A. Kurbatskii and R. Mankbadi, International Journal of Computational Fluid Dynamics, 18, 533-546 (2004).

[10] R. Liska and B. Wendroff, Composite Schemes for Conservation Laws, SIAM Journal on Numerical Analysis, 35, 2250-2271 (1998).

[11] D. P. Lockard, K. S. Brentner and H. L. Atkins, HighAccuracy Algorithms for Computational Aeroacoustics, AIAA Journal, 33, 246-251 (1995).

[12] W. De Roeck, W. Desmet, M. Baelmans and P. Sas, An Overview of High-Order Finite Difference Schemes for Computational Aeroacoustics, Proceedings of the International Conference on Noise and Vibration Engineering, Katholieke Universiteit Leuven, Belgium, 353-368 (2004).

[13] Takacs, A Two-Step Scheme for the Advection Equation with Minimized Dissipation and Dispersion Errors, Monthly Weather Review, 113, 1050-1065 (1985).

[14] C. K. W. Tam and J. C. Webb, Dispersion-RelationPreserving Finite Difference Schemes for Computational Acoustics, International Journal of Computational Physics, 107, 262-281 (1993).

[15] C. K. W. Tam, J. C. Webb and Z. Dong, A Study of the Short Wave Components in Computational Acoustics, Journal of Computational Acoustics, 1, 1-30 (1993).

[16] D. W. Zingg, H. Lomax and H. Jurgens, High-Accuracy Finite-Difference Schemes for Linear Wave Propagation, SIAM Journal on scientific Computing, 17, 328-346 (1996).

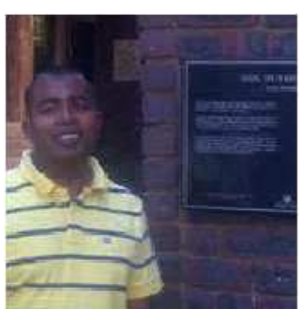

A. R. Appadu is a lecturer in the Department of Mathematics and Applied Mathematics at the University of Pretoria, South Africa. $\mathrm{He}$ received the $\mathrm{PhD}$ degree in Computational Fluid Dynamics at the University of Mauritius. His research interests are in the areas of Computational Fluid Dynamics, Computational Aeroacoustics and Optimisation. He has published research articles in reputed international journals in Applied Mathematics. $\mathrm{He}$ is referee of some international journals. 